\title{
A relationship between the force curve measured by atomic force microscopy in an ionic liquid and its density distribution on a substrate
}

\section{$\operatorname{AUTHOR}(S):$}

Amano, Ken-ichi; Yokota, Yasuyuki; Ichii, Takashi; Yoshida, Norio; Nishi, Naoya; Katakura, Seiji; Imanishi, Akihito; Fukui, Ken-ichi; Sakka, Tetsuo

\section{CITATION:}

Amano, Ken-ichi ... [et al]. A relationship between the force curve measured by ato mic force microscopy in an ionic liquid and its density distribution on a substrate. Physical Chemistry Chemical Physics 2017, 19(45): 30504-30512

\section{ISSUE DATE:}

2017-12-07

URL:

http://hdl.handle.net/2433/235736

\section{RIGHT:}

This is the accepted manuscript of the article, which has been published in final form at

https://doi.org/10.1039/c7cp06948k.; The full-text file will be made open to the public on 27 Oct 2018 in accordance with publisher's 'Terms and Conditions for Self-Archiving'.; この論文は出版社版でありません。引用の際には出版社版 をご確認ご利用ください。; This is not the published version. Please cite only the published version. 


\section{Graphical and Textual Abstract}

Relationship between the force curve measured in an ionic liquid and the solvation structure is studied. Applying the obtained relationship, candidates of the solvation structure are estimated from a measured force curve.
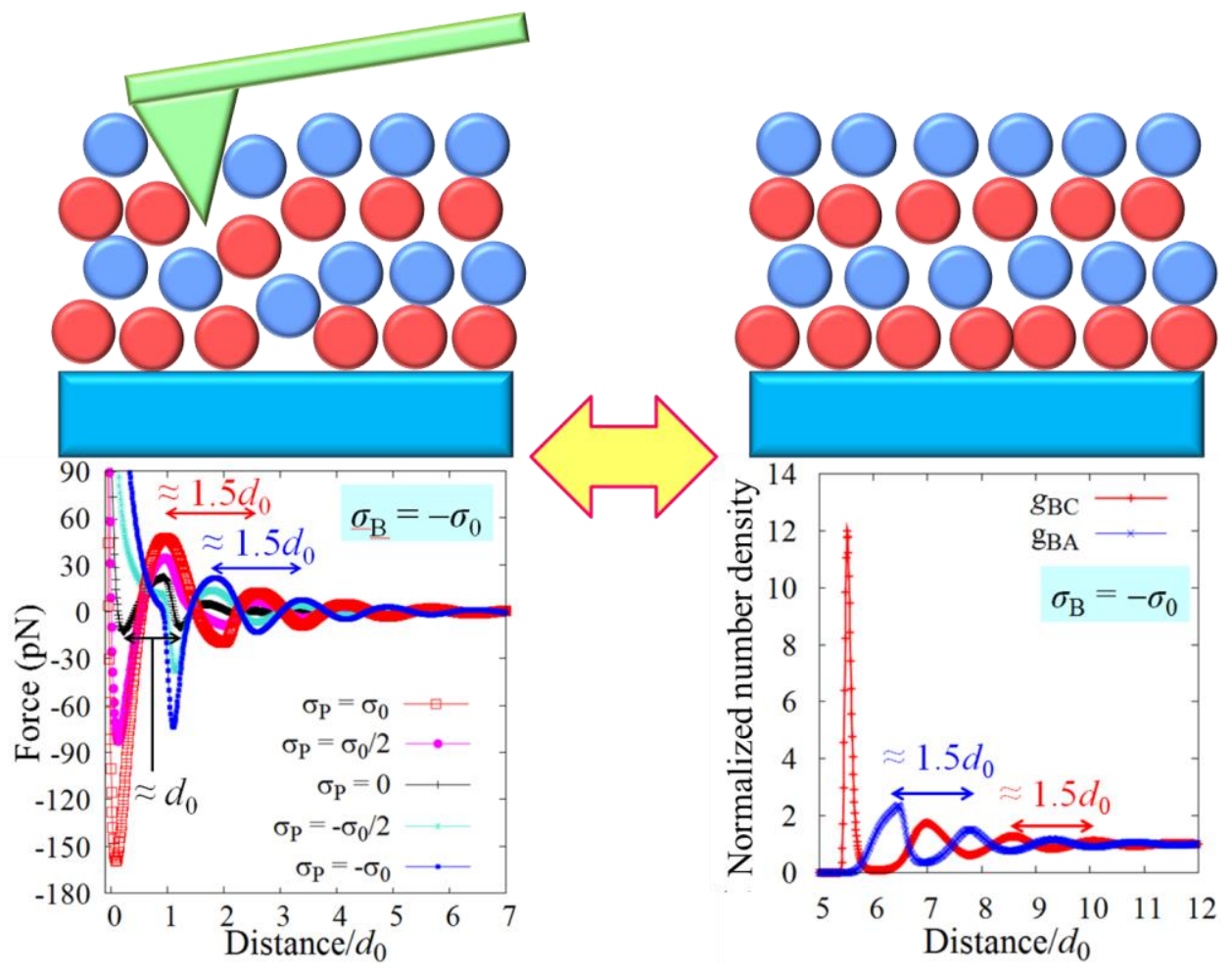


\section{Relationship between Force Curve Measured by Atomic}

\section{Force Microscopy in Ionic Liquid and its Density}

\section{Distribution on a Substrate}

Ken-ichi Amano, ${ }^{a *}$ Yasuyuki Yokota, ${ }^{b}$ Takashi Ichii, ${ }^{c}$ Norio Yoshida, ${ }^{d}$ Naoya Nishi, ${ }^{a}$ Seiji Katakura, ${ }^{a}$ Akihito Imanishi, ${ }^{e}$ Ken-ichi Fukui, ${ }^{e}$ and Tetsuo Sakka ${ }^{a}$

${ }^{a}$ Department of Energy and Hydrocarbon Chemistry, Graduate School of Engineering, Kyoto University, Kyoto 615-8510, Japan.

E-mail: amano.kenichi.8s@kyoto-u.ac.jp

${ }^{\mathrm{b}}$ Surface and Interface Science Laboratory, RIKEN, Saitama 351-0198, Japan

c Department of Materials Science and Engineering, Kyoto University, Kyoto, 606-8501, Japan

${ }^{\mathrm{d}}$ Department of Chemistry, Graduate School of Science, Kyushu University, Fukuoka 819-0395, Japan

e Department of Materials Engineering Science, Graduate School of Engineering Science, Osaka University, 1-3 Machikaneyama, Toyonaka, Osaka 560-8531, Japan

$\dagger$ Electronic supplementary information (ESI) available: Fig. S1 and Tables S1-S3 are included. 


\section{ABSTRACT}

An ionic liquid forms characteristic solvation structure on a substrate. For example, when the surface of the substrate is negatively or positively charged, the cation and anion layers are alternately aligned on the surface. Such solvation structure is closely related to slow diffusion, high electric capacity, and chemical reactions at the interface. To analyze the periodicity of the solvation structure, atomic force microscopy is often used. The measured force curve is generally oscillatory and its characteristic oscillation length corresponds not to ionic diameter, but to the ion-pair diameter. However, the force curve is not the solvation structure. Hence, it is necessary to know relationship between the force curve and the solvation structure. To find physical essences in the relationship, we have used statistical mechanics of a simple ionic liquid. We found that the basic relationship can be expressed by a simple equation and the reason why the oscillation length corresponds to the ion-pair diameter. Moreover, it is also found that Derjaguin approximation is applicable to the ionic liquid system. 


\section{Introduction}

Ionic liquids composed of cations and anions have great interests in many application areas. For example, they are used to enhance performances of $\operatorname{solar}^{1,2}$ and fuel cells. ${ }^{3}$ Since interfacial structures of the ionic liquids are closely related to slow diffusion, high electric capacity, and interfacial chemical reactions, the ionic liquids have valuable potential properties for batteries,,$^{4,5,6}$ capacitors, $^{7,8}$ and electrodeposition. ${ }^{9}$ To know the interfacial properties in more detail, density distributions of ionic liquids on substrates are studied theoretically and experimentally. Theoretical $^{10,11}$ and experimental ${ }^{12,13}$ studies have revealed that the ionic liquid forms layer structure on the substrate. For example, when the surface of the substrate is charged, the cation and anion layers are alternately aligned on the surface. ${ }^{13}$ Experimental studies by atomic force microscopy (AFM) ${ }^{14,15,16}$ and surface force apparatus (SFA) $)^{17,18}$ have also revealed such an interfacial property. AFM and SFA can measure force curves between (somewhat) arbitrary two surfaces in ionic liquids, and the force curves are basically oscillatory due to the layer structures of the ionic liquids. In addition, the oscillation length in the force curve generally corresponds to the ion-pair diameter, which is similar to that of the ionic liquid layer structure. The force curve reflects the layer structure of the ionic liquid, however, the force curve is not equal to the density distribution of the ionic liquid on the substrate. Therefore, elucidation of relationship between the force curve and the density distribution is important for properly analyzing the force curve.

Recent frequency modulation AFM (FM-AFM) can measure a force curve between a probe and a substrate in an ionic liquid without outstanding jumps (discontinuities) in the curve (see Fig. 1). ${ }^{16}$ In the experiment, the ionic liquid is 1-butyl-3-methylimidazolium bis(trifluoromethanesulfonyl)imide (BMIM-TFSI) and the substrate surface is rubrene (001). To avoid contamination of water, the ionic liquid 
was heated under vacuum at $120{ }^{\circ} \mathrm{C}$ for 4 hours, and it was stored in a closed bottle in a desiccator until use. In the force curve, the approach and retract curves coincide with each other (hysteresis is negligible). Hence, we can assume that the force curve was measured in equilibrium (reversible process). As shown in Fig. 1, the oscillation length of the force curve is close to the ion-pair diameter $(0.79 \mathrm{~nm}) .{ }^{19}$ This result is a general trend in the force curves in ionic liquids. ${ }^{14,15}$ From the force curve, one may imagine there is a layered structure of the ionic liquid on the substrate. However, the relationship between the force curve and the density distribution is not known in detail. Hence, we study the relationship between them from a theoretical point of view. In addition, we tackle on a question why the oscillation length of the force curve corresponds to the ion-pair diameter. In this study, we use statistical mechanics of a simple ionic liquid, ${ }^{20,21,22,23,24}$ where the cations and anions are modeled as small spheres with a point charge. The substrate and the probe are modeled as a relatively large sphere and an arbitrary sized sphere, respectively. To calculate the density distribution and the force curve, Ornstein-Zernike equation coupled with hypernetted chain closure $(\mathrm{OZ}-\mathrm{HNC})^{20,21,22,23,24}$ for a binary solvent is employed.

The relationship between the force curve and the density distribution has been studied by molecular dynamics (MD) simulations. ${ }^{25,26,27}$ Shape correlation between the force curve and the density distribution has been found by the MD simulations. In the paper, ${ }^{26}$ it is written that "there is certain lag between theoretical and computational studies of ionic liquids at charged interfaces and experimental works in this area" and "there is approximately 1 theoretical/computational publication per 50 experimental papers on this subject". Hence, we think more theoretical study of the ionic liquid on the interface should be done for connecting theoretical and experimental pictures. The MD simulations are important for searching the relationship, however, the simulations with the explicit molecular models make the analyses of the results difficult. That is, it 
is difficult to extract the physical essences from the complex model system. To find a fundamental relationship between the force curve and the density distribution, it is better to study the interfacial ionic liquid with a simplified model.

In this paper, we study the relationship using the simple spherical model. Introduction of the simple model enables us to find the relationship from a more theoretical point of view. Actually, it will be shown that the force curve and the density distribution can be connected by a simple equation. ${ }^{28,29}$ Thanks to the equation, a mechanism of the consistency between the oscillation length in the force curve and the ion-pair diameter will be explained. Furthermore, it will be found that Derjaguin approximation $^{30,31,32}$ is applicable also for the force curve measured in the ionic liquid.

In this paper, we study the relationship between the force curve measured in ionic liquid and its density distribution on the substrate on the basis of statistical mechanics of the simple ionic liquid. Recently, the force curves measured by AFM in ionic liquids are often discussed in this study area, however, the relationship between the force curve and the density distribution is not well known. Therefore, our work will contribute to this field, which indicates that analyses of the interfacial structure of the ionic liquid will be conducted more properly. Since studies of the interfacial structures are closely related to slow diffusion, high electric capacity, and chemical reactions at the interfaces, we believe the present work facilitates fundamental researches of such topics.

\section{Models and methods}

\section{Models in the ionic liquid system}

The models for the system of the ionic liquid are explained here. In the model, the ionic liquid is composed of an ensemble of small spheres. ${ }^{33}$ Two-body potential for the 
ionic liquid is expressed as

$$
u_{i j}\left(r_{i j}\right)=4 k_{\mathrm{B}} T\left(\frac{d_{0}}{r_{i j}}\right)^{12}+\frac{Z_{i} Z_{j} e^{2}}{4 \pi \varepsilon_{0} \varepsilon_{\mathrm{r}} r_{i j}},
$$

where the subscripts $i$ and $j$ represent cation or anion, $r_{i j}$ is the distance between the centers of the ion species $i$ and $j, k_{\mathrm{B}}$ is Boltzmann's constant, $T$ is the absolute temperature, $d_{0}$ corresponds to the diameter of the ion sphere which is set at $1 \mathrm{~nm}$. The diameters of the cation and anion are the same. $T$ is set at $300 \mathrm{~K}$, because the ionic liquid takes liquid state around at room temperature. The first term of the right-hand side in Eq. (1) represents the repulsive part of Lennard-Jones potential, while the second term represents the electrostatic potential, where $e, \varepsilon_{0}$, and $\varepsilon_{\mathrm{r}}$ represent elementary charge, electric permittivity in vacuum, and relative permittivity, respectively. $Z_{i}$ represents valence number of ion species $i$, the value of which is set at \pm 1 . To take into account the electric polarization effect, ${ }^{34,35,36}$ we set $\varepsilon_{\mathrm{r}}$ as $2 .^{33}$ The reduced bulk number densities $\left(\rho_{0} d_{0}{ }^{3}\right)$ for the ion species are both set at 0.3 , where $\rho_{0}$ is the bulk number density of cation or anion. (Generally, the reduced bulk number densities of cation and anion of a real ionic liquid are around 0.3.) The substrate and the probe are modelled as relatively large sphere and the arbitrary sized sphere, respectively. In this paper, we do not consider a double tip. Diameter of the spherical substrate $\left(d_{\mathrm{B}}\right)$ is set at $10 d_{0}$, and that of the spherical probe $\left(d_{\mathrm{P}}\right)$ is set at $d_{0}, 2 d_{0}, 3 d_{0}$, or $4 d_{0}$. Since the diameter of the cation corresponds to $d_{0}$ and its valence number is 1 , the surface charge density $\left(\sigma_{0}\right)$ can be estimated at $e /\left[4 \pi\left(d_{0} / 2\right)^{2}\right] \mathrm{C} / \mathrm{nm}^{2}$, and that of the anion is $-e /\left[4 \pi\left(d_{0} / 2\right)^{2}\right] \mathrm{C} / \mathrm{nm}^{2}$. Surface charge density of the spherical substrate $\left(\sigma_{\mathrm{B}}\right)$ is set as 0 or $-\sigma_{0}$, and that of the spherical probe $\left(\sigma_{\mathrm{P}}\right)$ is set as $0, \pm \sigma_{0} / 2$, or $\pm \sigma_{0}$. Three two-body potentials between the spherical substrate-ion, the spherical probe-ion, and the spherical substrate-spherical probe are expressed as 


$$
u_{i j}\left(r_{i j}\right)=4 k_{\mathrm{B}} T\left(\frac{d_{0}}{r_{i j}-\left(d_{i}+d_{j}\right) / 2+d_{0}}\right)^{12}+\frac{\pi^{2} \sigma_{i} \sigma_{j} d_{i}^{2} d_{j}^{2}}{4 \pi \varepsilon_{0} \varepsilon_{\mathrm{r}} r_{i j}}
$$

where the subscript $i$ and $j$ represent the spherical substrate, spherical probe, cation, or anion. $r_{i j}$ is the distance between the centers of the species $i$ and $j$.

\section{Calculations of the ionic liquid structure and the force curve}

To calculate the density distribution of the ionic liquid on the substrate and the force curve, we wrote a following program. In the program, we employed an integral equation theory (OZ-HNC) for a simple ionic liquid. ${ }^{20,21,22,23,24}$ The OZ-HNC is known as a precise calculation method for a long range interaction system. To sufficiently converge the numerical calculation, we used Picard iteration combined with temperature annealing ${ }^{20}$ and modified direct inversion in the iterative subspace (MDIIS). ${ }^{37}$ To perform the calculation without cut off of the tail of the electrostatic interaction, we used a renormalization method. ${ }^{37}$ First, the bulk structure of the ionic liquid is calculated. Second, the number density distributions of the cations and anions on the substrate and the probe are calculated by using the previously obtained bulk structures. Finally, the force curve between the substrate and the probe is calculated by using the density distributions of the cations and anions on the substrate and the probe (see Fig. $\mathrm{S}^{\dagger}$ ). In all of the calculations above, the absolute temperature is set at $300 \mathrm{~K}$. 


\section{Results and discussion \\ Density distribution of the ionic liquid}

There are two models of the substrates, one has no surface charge density $\left(\sigma_{\mathrm{B}}=0\right)$, and the other has it $\left(\sigma_{\mathrm{B}}=-\sigma_{0}\right)$. When $\sigma_{\mathrm{B}}=0$, the density distributions of the cation and anion are the same (Fig. 2(a)). The layer-to-layer distance is almost equal to $d_{0}$, which is not equal to this model's ion-pair diameter $\approx 1.5 d_{0}{ }^{38}$ Meanwhile, when $\sigma_{\mathrm{B}}=-\sigma_{0}$, the density distributions of the cation and anion are different from each other, and their layers are alternately aligned (Fig. 2(b)). Because the substrate surface is negatively charged, the first layer is composed of the cations, and the second layer is composed of the anions. As shown in Fig. 2(b), the layer-to-layer distances of the cation and anion are both $\approx 1.5 d_{0}$, which is corresponding to this model's ion-pair diameter. ${ }^{38}$ In other words, there is consistency between the layer-to-layer distance and the ion-pair diameter.

\section{Force curve calculated by the ideal probe}

In AFM experiments, effective diameter of the tip apex of the probe is supposed to be $\mathrm{nm}$ scale ${ }^{39,40}$ because molecular-scale topographies of the crystal surfaces can be measured. ${ }^{15,16}$ For that reason, we introduced nm-scale model probes with $d_{\mathrm{P}}=d_{0}, 2 d_{0}$, $3 d_{0}$, and $4 d_{0}\left(d_{0}=1 \mathrm{~nm}\right)$ in the model section above. For elucidation of the (simple) relationship between the force curve and the density distribution, Amano et al. ${ }^{28}$ have considered a binary solvent composed of two types of spheres and approximated the probe as one solvent molecule (i.e., cation or anion). Here we mention the solvent probe as the ideal probe. When the ideal probe is the same as a cation it is called the ideal cationic probe, while when it is an anion it is called the ideal anionic probe. In 
the simplified model, the relationship between the force curve and the density distribution is exactly derived: $:^{28}$

$$
f_{\mathrm{BP} i}\left(r_{\mathrm{B} i}\right)=k_{\mathrm{B}} T \frac{\partial \ln g_{\mathrm{B} i}\left(r_{\mathrm{B} i}\right)}{\partial r_{\mathrm{B} i}}
$$

where $i$ represents cation (c) or anion (a), $f_{\mathrm{BP} i}$ is the force curve between the substrate and the ideal cationic or anionic probe, $g_{\mathrm{B} i}$ is the normalized number density distribution of the cation or anion on the substrate, $r_{\mathrm{B} i}$ is distance between the substrate and the cation (ideal cationic probe) or anion (ideal anionic probe). From Eq. (3), we can briefly understand that when gradient of $g_{\mathrm{B} i}$ is positive $f_{\mathrm{BP} i}$ is also positive (repulsive force), while when gradient of $g_{\mathrm{B} i}$ is negative $f_{\mathrm{BP} i}$ is also negative (attractive force). Interestingly, the right-hand side of Eq. (3) contains the density distribution of the only one component (cation or anion). Thus, when the ideal probe is cationic, its force curve has only information of cation density distribution, and vice versa.

In Fig. 3, force curves for the ideal cationic and anionic probes are shown. The solvation forces are calculated by substituting the density distributions of cation and anion on the substrate and around the ideal probes (the four distributions are substituted) into the OZ-HNC (see Fig. $\mathrm{S}^{\dagger}$ ). That is, Eq. (3) is not used here. However, we could perfectly reconstruct the force curves in Fig. 3 from Fig. 2 through Eq. (3) (not shown, because there are no differences). It is an evidence of the validity of Eq. (3).

By comparing Fig. 1 and Fig. 3, it is found that the magnitudes of the force amplitudes are consistent with each other. It indicates that the effective diameter of the tip apex of the real probe is also $\mathrm{nm}$ scale, because if the effective diameter is much larger than that scale, the force amplitude is supposed to be much larger than that of the ideal probe. To the best of our knowledge, ${ }^{25}$ consistency between the theoretical 
and experimental force amplitudes is confirmed for the first time in the case of ionic-liquid AFM. (There could be a possibility that the solvation structure of BMIM-TFSI on the rubrene (001) surface is less structured in contrast to a solvation structure of an ionic liquid on an inorganic solid surface. ${ }^{41}$ )

Here, using Eq. (3), we simply explain the reason why the oscillation length of the real force curve generally corresponds to the ion-pair diameter. In the experimental condition (the real case), surfaces of the substrate and the probe are generally charged. In that case, the density distribution of the ionic liquid on the substrate takes the alternately aligned layer structure like in Fig. 2(b). Such density distributions $\left(g_{\text {Bc }}\right.$ and $\left.g_{\mathrm{Ba}}\right)$ can be transformed into the force curves for the ideal cationic $\left(f_{\mathrm{BPc}}\right)$ and anionic $\left(f_{\mathrm{BPa}}\right)$ probes, respectively, using Eq. (3). Because the $g_{\mathrm{Bc}}$ and $g_{\mathrm{Ba}}$ have the oscillation length of the ion-pair diameter, $f_{\mathrm{BPc}}$ and $f_{\mathrm{BPa}}$ calculated from Eq. (3) also have almost the same oscillation length.

We do not know the diameter of the real tip apex, however, its effective diameter should be nm scale. ${ }^{15,16,39,40}$ Moreover, the real probe surface should be charged, due to ionization (polarization) of the surface. In that case, the real probe is close to the ideal probe. Thus, shape of the real force curve may be similar to that obtained from the ideal probe $\left(f_{\mathrm{BPc}}\right.$ or $\left.f_{\mathrm{BPa}}\right)$. Therefore, the force curves measured by $\mathrm{AFM}$ in ionic liquids generally have the ion-pair diameter in the oscillation length.

\section{Effect of surface charge density of the probe}

In this section, we conduct a parameter survey of the surface charge density $\sigma_{\mathrm{P}}$, where diameter of the probe $d_{\mathrm{P}}$ is fixed at $2 d_{0}$ for simplicity. Fig. 4 shows the force curves for several $\sigma_{\mathrm{P}}$. In Fig. 4(a), the surface charge density of the substrate $\sigma_{\mathrm{B}}$ is 0 . In 
this case, the oscillation lengths of the force curves are $\approx d_{0}$. As $\left|\sigma_{\mathrm{P}}\right|$ decreases to zero, the minimum value of the force decreases (see force curves around Distance $/ d_{0} \approx 0$ ). The decrease in $\left|\sigma_{\mathrm{P}}\right|$ means increase in the solvophobicity leading to increase of the solvophobic (attractive) interaction. Therefore, when the substrate is solvophobic, adhesion force increases with decreasing $\left|\sigma_{\mathrm{P}}\right|$.

Fig. 4(b) shows the force curves in the case of the charged substrate $\left(\sigma_{\mathrm{B}}=-\sigma_{0}\right)$. Since the substrate is negatively charged, the minimum value in the force curve decreases as $\sigma_{\mathrm{P}}$ increases from 0 to $\sigma_{0}$ (see force curves around Distance $/ d_{0} \approx 0$ ). Interestingly, the minimum value also decreases as $\sigma_{\mathrm{P}}$ decreases from 0 to $-\sigma_{0}$ (see force curves around Distance/ $d_{0} \approx 1$ ). This attractive force can be explained by the counter ions confined between them. ${ }^{17,18}$ As shown in Fig. 4(b), when the probe is charged, the oscillation length of the force curve is $\approx 1.5 d_{0}$. On the other hand, when the probe is uncharged (neutral), the oscillation length is $\approx d_{0}$. That is, when either the substrate or the probe is neutral, the oscillation length does not correspond to the ion-pair diameter.

\section{Effect of diameter of the probe}

In this section, we conduct a parameter survey of the probe diameter $d_{\mathrm{P}}$, where $\left|\sigma_{\mathrm{P}}\right|$ of the probe surface charge density is fixed at $\sigma_{0}$ for simplicity. Fig. 5 shows the force curve on the neutral substrate $\left(\sigma_{\mathrm{B}}=0\right)$. It is found that as $d_{\mathrm{P}}$ increases, the amplitude of the force curve increases. The reason is as follows: when the probe diameter is large, the solvation structure destructed by the probe is also large leading to the increase in the force amplitude.

Fig. 6(a) shows the force curves between the negatively charged substrate surface 
and the positively charged probes. Meanwhile in Fig. 6(b), the substrate and probe surfaces are both negatively charged. Also in these cases, the force amplitudes increase as $d_{\mathrm{P}}$ increases.

\section{Derjaguin approximation in the ionic liquid}

From Figs. 5 and 6, we can realize that the overall shapes of the force curves of the non-ideal probes are similar to that of the ideal probes. Thus, it is expected that the force curve of the non-ideal probe can mimic that of the ideal probe through a scaling operation. In this section, we check the validity of the expectation and discuss the applicability of Derjaguin approximation ${ }^{30,31,32}$ in the ionic liquid. Derjaguin approximation is famous approximation in research fields of AFM and SFA, which can connect the force curve between the arbitrarily shaped substances (e.g., sphere, cylinder, planer wall) and the potential per unit area between two-planner walls $(W)$. In the present situation, Derjaguin approximation can be given by ${ }^{31,42}$

$$
f_{\mathrm{BP}}(h)=\pi W(h) \frac{\left(d_{\mathrm{B}}+t_{\mathrm{B}}\right)\left(d_{\mathrm{P}}+t_{\mathrm{P}}\right)}{\left(d_{\mathrm{B}}+t_{\mathrm{B}}\right)+\left(d_{\mathrm{P}}+t_{\mathrm{P}}\right)},
$$

where $f_{\mathrm{BP}}$ is force between the substrate and the probe, $h$ is nearest neighbour distance between the focusing surfaces, $t_{\mathrm{B}}$ and $t_{\mathrm{P}}$ represent additive thicknesses for the substrate and the probe, respectively. (When solvent is hard-sphere fluid, the both thicknesses are equal to the radius of the hard sphere.) In the case of the ionic liquid, exact evaluation of the additive thickness is difficult. However, Eq. (4) can be reduced to $f_{\mathrm{BP}}(h)=\pi W(h) C_{\mathrm{BP}}$, where $C_{\mathrm{BP}}$ is a coefficient depending on properties of the substrate and the probe. Similarly, for the ideal cationic or anionic probe, the Derjaguin 
approximation can be written as $f_{\mathrm{BP} i}(h)=\pi W(h) C_{\mathrm{BP} i}$, where $i$ represent cation (c) or anion (a), and $C_{\mathrm{BP} i}$ is also the coefficient. Hence, the force curves for the ideal and non-ideal probes can be connected as follows:

$$
f_{\mathrm{BP} i}(h)=C^{*} f_{\mathrm{BP}}(h),
$$

where $C^{*}$ is also the coefficient. Generally, $C^{*}$ is smaller than or equal to 1 , because the effective diameter of the real tip apex is normally larger than that of the ideal cationic or anionic probe. Deviation from 1 means size and/or surface charge deviations from the ideal probe. We notify that Eq. (5) is derived in an implicit condition that $\sigma_{\mathrm{B}}$ in $f_{\mathrm{BP}}$ and $\sigma_{\mathrm{B}}$ in $f_{\mathrm{BP} i}$ are the same and $\sigma_{\mathrm{P}}=\sigma_{0}$. Eq. (5) indicates the force curve of the non-ideal probe can be changed to that of the ideal probe simply by the scaling. That is, if Eq. (5) is applicable in the ionic liquid, the number density distribution of the ionic liquid on the substrate can be estimated by also using Eq. (3).

Figs. 7 and 8 show the results of the scaling. The scaling was performed so that the minimum value of the force curve of the non-ideal probe matches that of the ideal probe. We found that the scaling values $\left(C^{*}\right)$ are ranged from about 0.2 to 0.9 (see Tables $\left.\mathrm{S} 1-\mathrm{S} 3^{\dagger}\right)$. Since difference between the ideal force curve and the scaled ideal force curve is small compared with that without the scaling, the Derjaguin approximation is applicable also in the ionic liquid system. Especially, the difference is much smaller in the cases of the charged substrate surface (Fig. 8).

The force curves for the probes with $\sigma_{\mathrm{P}}= \pm \sigma_{0} / 2$ are also scaled in Figs. 7 and 8. Although $\sigma_{\mathrm{P}}$ is not equal to $\sigma_{0}$ in the situation, difference between the ideal force curve and the scaled ideal force curve is not relatively large. This feature is convenient for us, because in the experiment it is difficult to prepare a probe that has the same surface charge density as that of the ideal probe. Using this feature, it is possible to roughly 
estimate the scaled ideal force curve from an experimentally obtained force curve. Subsequently, the density distribution of the ionic liquid on the substrate can be estimated by using Eq. (3). However, when we estimate it from the experimental data, we should know or empirically set the value of $C^{*}$. We notify that the hysteresis of the measured force curve should be sufficiently small, when this kind of the operation is performed, because Eq. (3) is a relationship in equilibrium.

Since we found that the Derjaguin approximation is applicable in the ionic liquid, we try estimating the density distribution of the ionic liquid on the substrate from Figs. 7 and 8 by using Eq. (3). Figs. 9, 10(a), and 10(b) show the density distributions estimated from Figs. 7, 8(a), and 8(b), respectively. From Fig. 10(b), it is found that when the surfaces of the substrate and the probe are both negatively charged, the density distributions are relatively well estimated. Also when the surface charges of the substrate and the probe are opposite (Fig. 10(a)), the deviations from the right density distribution are not so large. When the substrate is neutral (Fig. 9), the deviations are relatively large. However, the layer-to-layer distances are well predicted.

\section{Candidates of the surface density distribution of $\mathrm{TFSI}^{-}$}

As a trial, we transform the experimentally obtained force curve (Fig. 1) at $300 \mathrm{~K}$ into the number density distribution by using both Eq. (3) and Eq. (5), where $d_{\mathrm{B}}$ is set as $\infty$. In the experiment, ${ }^{16}$ the ionic liquid is BMIM-TFSI, and the substrate surface is rubrene (001). Fig. 11 shows candidates of the number density distribution of the anion $\left(\mathrm{TFSI}^{-}\right)$on the rubrene $(001)$ surface, where $C^{*}$ is empirically set at $1,0.75,0.5$, or 0.25. Although $C^{*}$ is empirically set in the estimation, we believe that the qualitatively 
proper density distribution exists in Fig. 11.

As stated above, Fig. 11 shows the anion number density distribution. Reasons of the anion are explained as follows. In the experiment, ${ }^{16}$ a silicon cantilever was used, and hence its probe surface is considered to be negatively charged due to $\mathrm{SiO}^{-}{ }^{43}$ Moreover, molecular-scale topography of the crystal surface was measured by the same probe, which indicates that the effective diameter of its tip apex was also very small. Then, the probe can be roughly hypothesized as an ideal anionic probe. In such a case, the density distribution estimated by the present method (combination of Eq. (3) and Eq. (5)) becomes that of the anion.

Next, we argue about the shoulder pointed by the arrow (see Fig. 11). From height of the shoulder, it is thought that the measured rubrene surface is also negatively charged. Although it is mysterious for the rubrene, an expected mechanism of the negative charge is as follows. When the force curve was measured, we put the rubrene crystal on a glass sheet. In the preparation of the substrate, we found adhesion force between the rubrene crystal and the glass sheet when they contacts. It is considered that the adhesion force attributes to electrostatic interaction between them, where the glass surface is negative and the rubrene surface is positive. This is because the glass sheet surface tends to be negatively charged against various substances. In a normal condition, the rubrene crystal is insulator, and thus the opposite surface of the rubrene (rubrene | ionic liquid interface) is assumed to be negatively charged due to the dielectric polarization. Consequently, in the experimental condition, it is expected that the low density of anion at the arrowed position (Fig. 11) originates from the negatively charged rubrene surface at the rubrene | ionic liquid interface. 


\section{Conclusions}

We have studied the relationship between the force curve in the ionic liquid measured by AFM and the density distribution of the ionic liquid on the substrate. It has been found that when either the substrate or the probe is uncharged, the oscillation length of the force curve does not correspond to the ion-pair diameter, but the diameter of the anion or cation. Meanwhile, when the substrate and the probe are both charged, the oscillation length of the force curve corresponds to the ion-pair diameter. That is, when the oscillation length of the experimental force curve corresponds to the ion-pair diameter, both surfaces of the substrate and the probe may be charged. The reason of the consistency between the oscillation length in the experimental force curve and the ion-pair diameter has been explained by using Eq. (3). A number of the force curves of the non-ideal probes have been calculated, and we found that they fairly obey Derjaguin approximation even in the ionic liquid. By using Eq. (3) and the Derjaguin approximation (Eq. (5)), we have obtained the candidates of the number density distributions of the $\mathrm{TFSI}^{-}$on the rubrene (001) surface. Although it is an approximative method, the density distribution of the ionic liquid on the substrate is obtained from the experimental data for the first time in the study field of the ionic-liquid AFM. In summary, we have elucidated the fundamental relationship between the force curve and the density distribution in the ionic liquid. The model system we introduced is relatively simple, however, it is considered that being simple is important for extracting the physical essence in the system. This study will fill a gap between the theoretical/computational studies of ionic liquids at the interface and that of experimental studies. The findings will be helpful for understanding an interaction between solutes (colloidal particles) in an ionic liquid from viewpoint of the solvation structure. In the future, we would like to perform comparison between a force curve measured in an ionic colloidal dispersion system and the density distribution of the 
ionic colloidal particles as a sequential study.

\section{Conflicts of interest}

There are no conflicts to declare.

\section{Acknowledgements}

We thank Hiroshi Onishi for comments and facilitating the cooperation on this study. We appreciate Hirofumi Sato's advice on MDIIS. This work was mainly supported by Grant-in-Aid (15K21100) for Young Scientists (B) from Japan Society for the Promotion of Science (JSPS), and partially supported by Grant-in-Aid (26410092) for Scientific Research (C) from JSPS, Funding Program for Next Generation World-Leading Researchers (GR071) from JSPS, and JSPS Bilateral Open Partnership Joint Research Projects.

\section{References}

1 G. P. Salvador, D. Pugliese, F. Bella, A. Chiappone, A. Sacco, S. Bianco and M. Quaglio, New insights in long-term photovoltaic performance characterization of cellulose-based gel electrolytes for stable dye-sensitized solar cells, Electrochim. Acta, 2014, 146, 44-51. 
J. R. Nair, L. Porcarelli, F. Bella and C. Gerbaldi, Newly Elaborated

Multipurpose Polymer Electrolyte Encompassing RTILs for Smart

Energy-Efficient Devices, ACS Appl. Mater. Interfaces, 2015, 7, 12961-12971.

3 F. Liu, S. Wang, J. Li, X. Tian, X. Wang, H. Chen and Z. Wang,

Polybenzimidazole/ionic-liquid-functional silica composite membranes with improved proton conductivity for high temperature proton exchange membrane fuel cells, J. Memb. Sci., 2017, 541, 492-499.

4 H. Nakagawa, S. Izuchi, K. Kuwana, T. Nukuda and Y. Aihara, Liquid and Polymer Gel Electrolytes for Lithium Batteries Composed of Room-Temperature Molten Salt Doped by Lithium Salt, J. Electrochem. Soc., 2003, 150, A695.

5 M. Yamagata, Y. Matsui, T. Sugimoto, M. Kikuta, T. Higashizaki, M. Kono and M. Ishikawa, High-performance graphite negative electrode in a bis(fluorosulfonyl)imide- based ionic liquid, J. Power Sources, 2013, 227, 6064.

6 A. Basile, A. I. Bhatt and A. P. O'Mullane, Stabilizing lithium metal using ionic liquids for long-lived batteries, Nat. Commun., 2016, 7, 1-11.

7 T. Sato, G. Masuda and K. Takagi, Electrochemical properties of novel ionic liquids for electric double layer capacitor applications, Electrochim. Acta, 2004, 49, 3603-3611.

8 N. Fechler, T. P. Fellinger and M. Antonietti, 'salt templating': A simple and sustainable pathway toward highly porous functional carbons from ionic liquids, Adv. Mater., 2013, 25, 75-79.

9 S. Zein El Abedin, E. M. Moustafa, R. Hempelmann, H. Natter and F. Endres, Electrodeposition of nano- and macrocrystalline aluminium in three different air and water stable ionic liquids, ChemPhysChem, 2006, 7, 1535-1543.

10 E. Paek, a. J. Pak and G. S. Hwang, A computational study of the interfacial 
structure and capacitance of graphene in [BMIM][PF6] ionic liquid, $J$.

Electrochem. Soc., 2012, 160, A1-A10.

11 Y.-L. Wang, Z.-Y. Lu and A. Laaksonen, Heterogeneous dynamics of ionic liquids in confined films with varied film thickness., Phys. Chem. Chem. Phys., 2014, 16, 20731-40.

12 H. Zhou, M. Rouha, G. Feng, S. S. Lee, H. Docherty, P. Fenter, P. T. Cummings, P. F. Fulvio, S. Dai, J. Mcdonough, V. Presser and Y. Gogotsi, Nanoscale Perturbations of Room Temperature Ionic Liquid Structure at Charged and Uncharged Interfaces.pdf, ACS Nano, 2012, 9818-9827.

13 M. Mezger, H. Shiröder, H. Reichert, S. Schramm, J. S. Okasinski, S. Schöder, V. Honkimäki, M. Deutsch, B. M. Ocko, J. Ralston, M. Rohwerder, M.

Stratmann and H. Dosch, Molecular Layering of Fluorinated, Sicence, 2008, 322, $424-428$.

14 R. Atkin and G. G. Warr, Structure in confined room-temperature ionic liquids, J. Phys. Chem. C, 2007, 111, 5162-5168.

15 T. Ichii, M. Negami, M. Fujimura, K. Murase and H. Sugimura, Structural Analysis of Ionic-liquid/Organic-monolayer Interface by Phase Modulation Atomic Force Microscopy Utilizing a Quartz Tuning Fork Sensor, Electrochemistry, 2014, 82, 380-384.

16 Y. Yokota, H. Hara, Y. Morino, K. Bando, A. Imanishi, T. Uemura, J. Takeya and K. Fukui, Molecularly clean ionic liquid/rubrene single-crystal interfaces revealed by frequency modulation atomic force microscopy., Phys. Chem. Chem. Phys., 2015, 17, 6794-6800.

17 S. Perkin, Ionic liquids in confined geometries, Phys. Chem. Chem. Phys., 2012, 14, 5052 .

18 A. M. Smith and S. Perkin, Influence of Lithium Solutes on Double-Layer 
Structure of Ionic Liquids, J. Phys. Chem. Lett., 2015, 6, 4857-4861.

Y. Yokota, T. Harada and K. Fukui, Direct observation of layered structures at ionic liquid/solid interfaces by using frequency-modulation atomic force microscopy., Chem. Commun., 2010, 46, 8627-8629.

20 J. P. Hansen and I. R. McDonald, Statistical mechanics of dense ionized matter. IV. Density and charge fluctuations in a simple molten salt, Phys. Rev. A, 1975, 11, 2111-2123.

21 B. Larsen, Studies in statistical mechanics of Coulombic systems. II. Ergodic problems in Monte Carlo simulations of the restricted primitive model, J. Chem. Phys., 1978, 68, 4511-4523.

22 M. Kinoshita, M. Harada and A. Shioi, Characteristics of solutions of the HNC equation applied to anion-cation systems interacting through a strong long-range Coulomb potential, Molcular Phys., 1990, 70, 1121-1134.

23 M. J. Booth and a. D. J. Haymet, Molten salts near a charged surface: integral equation approximation for a model of KCl, Mol. Phys., 2001, 99, 1817-1824.

24 J.-P. Hansen and I. R. McDonald, Theory of Simple Liquids, Academic Press, London, 3rd ed., 2006.

25 M. V. Fedorov and R. M. Lynden-Bell, Probing the neutral graphene-ionic liquid interface: insights from molecular dynamics simulations, Phys. Chem. Chem. Phys., 2012, 14, 2552.

26 V. Ivaništšev, M. V. Fedorov and R. M. Lynden-Bell, Screening of Ion Graphene Electrode Interactions by Ionic Liquids: The Effects of Liquid Structure, J. Phys. Chem. C, 2014, 118, 5841-5847.

27 M. V Fedorov and A. A. Kornyshev, Ionic Liquids at Electrified Interfaces, Chem. Rev., 2014, 114, 2978-3036.

28 K. Amano, K. Suzuki, T. Fukuma and H. Onishi, Relation between a force curve 
measured on a solvated surface and the solvation structure: Relational expressions for a binary solvent and a molecular liquid, arXiv, 2012, 1212.6138, $1-20$.

29 K. Amano, K. Suzuki, T. Fukuma, O. Takahashi and H. Onishi, The relationship between local liquid density and force applied on a tip of atomic force microscope: A theoretical analysis for simple liquids, J. Chem. Phys., 2013, 139, 224710.

30 B. V. Derjaguin, Y. I. Rabinovich and N. V. Churaev, Measurement of forces of molecular attraction of crossed fibres as a function of width of air gap, Nature, 1977, 265, 520-521.

31 M. Oettel, Depletion force between two large spheres suspended in a bath of small spheres: Onset of the Derjaguin limit, Phys. Rev. E, 2004, 69, 41404.

32 S. Rentsch, R. Pericet-camara, G. Papastavrou and M. Borkovec, Probing the validity of the Derjaguin approximation for heterogeneous colloidal particles, Phys. Chem. Chem. Phys., 2006, 8, 2531-2538.

33 M. V. Fedorov and A. A. Kornyshev, Towards understanding the structure and capacitance of electrical double layer in ionic liquids, Electrochim. Acta, 2008, 53, 6835-6840.

34 I. Leontyev and A. Stuchebrukhov, Accounting for electronic polarization in non-polarizable force fields, Phys. Chem. Chem. Phys., 2011, 13, 2613.

35 F. Dommert, K. Wendler, R. Berger, L. Delle Site and C. Holm, Force fields for studying the structure and dynamics of ionic liquids: A critical review of recent developments, ChemPhysChem, 2012, 13, 1625-1637.

36 R. Ishizuka and N. Matubayasi, Self-Consistent Determination of Atomic Charges of Ionic Liquid through a Combination of Molecular Dynamics Simulation and Density Functional Theory, J. Chem. Theory Comput., 2016, 12, 
804-811.

37 A. Kovalenko, S. Ten-no and F. Hirata, Solution of three-dimensional reference interaction site model and hypernetted chain equations for simple point charge water by modified method of direct inversion in iterative subspace, J. Comput. Chem., 1999, 20, 928-936.

38 L. Jones and P. Atkins, Chemistry: Molecules, Matter and Change, W. H. Freeman \& Co Ltd, 4th ed., 2000.

39 T. Hiasa, K. Kimura and H. Onishi, Minitips in frequency-modulation atomic force microscopy at liquid-solid interfaces, Jpn. J. Appl. Phys., 2012, 51, 25703.

40 K. Amano, Y. Liang, K. Miyazawa, K. Kobayashi, K. Hashimoto, K. Fukami, N. Nishi, T. Sakka, H. Onishi and T. Fukuma, Number density distribution of solvent molecules on a substrate: a transform theory for atomic force microscopy, Phys. Chem. Chem. Phys., 2016, 18, 15534-15544.

41 K. Fukui, Y. Yokota and A. Imanishi, Local Analyses of Ionic Liquid/Solid Interfaces by Frequency Modulation Atomic Force Microscopy and Photoemission Spectroscopy, Chem. Rec., 2014, 14, 964-973.

42 K. Amano, M. Iwaki, K. Hashimoto, K. Fukami, N. Nishi, O. Takahashi and T. Sakka, Number Density Distribution of Small Particles around a Large Particle: Structural Analysis of a Colloidal Suspension, Langmuir, 2016, 32, $11063-11070$.

43 S. M. R. Akrami, H. Nakayachi, T. Watanabe-Nakayama, H. Asakawa and T. Fukuma, Significant improvements in stability and reproducibility of atomic-scale atomic force microscopy in liquid., Nanotechnology, 2014, 25, 455701. 


\section{Figure captions}

Fig. 1 A force curve measured by Yokota (the second author) et al. using FM-AFM, where the ionic liquid is BMIM-TFSI and the substrate surface is rubrene (001) surface. ${ }^{16}$ The ion-pair diameter of BMIM-TFSI liquid is $0.79 \mathrm{~nm} .{ }^{19}$

Fig. 2 The normalized number density distributions of the cations and the anions on the substrate calculated by the OZ-HNC. Surface charge density of the substrate $\sigma_{\mathrm{B}}$ is 0 in (a) and $\sigma_{\mathrm{B}}$ is $-\sigma_{0}$ in (b).

Fig. 3 Force curve between the substrate and the ideal cationic (anionic) probe calculated by the OZ-HNC. $\sigma_{\mathrm{B}}$ is 0 in (a) and $\sigma_{\mathrm{B}}$ is $-\sigma_{0}$ in (b). Since the probe is the ideal one, $d_{\mathrm{P}}=d_{0}$.

Fig. 4 Force curve between the substrate and the probe with $d_{\mathrm{P}}=2 d_{0}$ calculated by the OZ-HNC. $\sigma_{\mathrm{B}}$ is 0 in (a) and $\sigma_{\mathrm{B}}$ is $-\sigma_{0}$ in (b).

Fig. 5 Force curve between the neutral substrate and the positively charged probe calculated by the OZ-HNC. When $d_{\mathrm{P}}=d_{0}$, the force curve is that of the ideal cationic or anionic probe.

Fig. 6 Force curve between the negatively charged substrate and the charged probe 
calculated by the OZ-HNC. $\sigma_{\mathrm{P}}=\sigma_{0}$ in (a) and $\sigma_{\mathrm{P}}=-\sigma_{0}$ in (b). When $d_{\mathrm{P}}=d_{0}$, the force curve is that of the ideal cationic or anionic probe.

Fig. 7 The scaled force curve between the neutral substrate and the charged probe. The purple force curve is the benchmark, which is that of the ideal cationic or anionic probe. Each scaling value $C^{*}$ is written in Table $\mathrm{S} 1 .^{\dagger}$

Fig. 8 The scaled force curve between the charged substrate and the charged probe. In (a) and (b), the surface charge of the probes are positive and negative, respectively. The purple force curves are the benchmarks, which are the force curves of the ideal cationic and anionic probes. Scaling $C^{*}$ values in (a) and (b) are written in Tables S2 and $S 3,{ }^{\dagger}$ respectively.

Fig. 9 The normalized number density distributions of the cations (or anions) on the neutral substrate. They are calculated from the force curves in Fig. 7. The benchmark structure is colored in purple. The density distributions of the cations and anions are the same due to the neutrality of the substrate surface.

Fig. 10 The normalized number density distributions of the cations (a) and the anions (b) on the negatively charged substrate, which are calculated from the force curves in Figs. 8(a) and 8(b), respectively. The benchmark structures are colored in purple. 
Fig. 11 Candidates of the density distributions of the $\mathrm{TFSI}^{-}$on the rubrene (001) surface calculated from the experimentally obtained force curve (Fig. 1). The rubrene surface is considered to be negatively charged due to the dielectric polarization. The black solid line $\left(C^{*}=1\right)$, the red dotted line $\left(C^{*}=0.75\right)$, the green short broken line $\left(C^{*}\right.$ $=0.5)$, the purple long broken line $\left(C^{*}=0.25\right)$. The ion-pair diameter of BMIM-TFSI liquid is $0.79 \mathrm{~nm}^{19}$ 


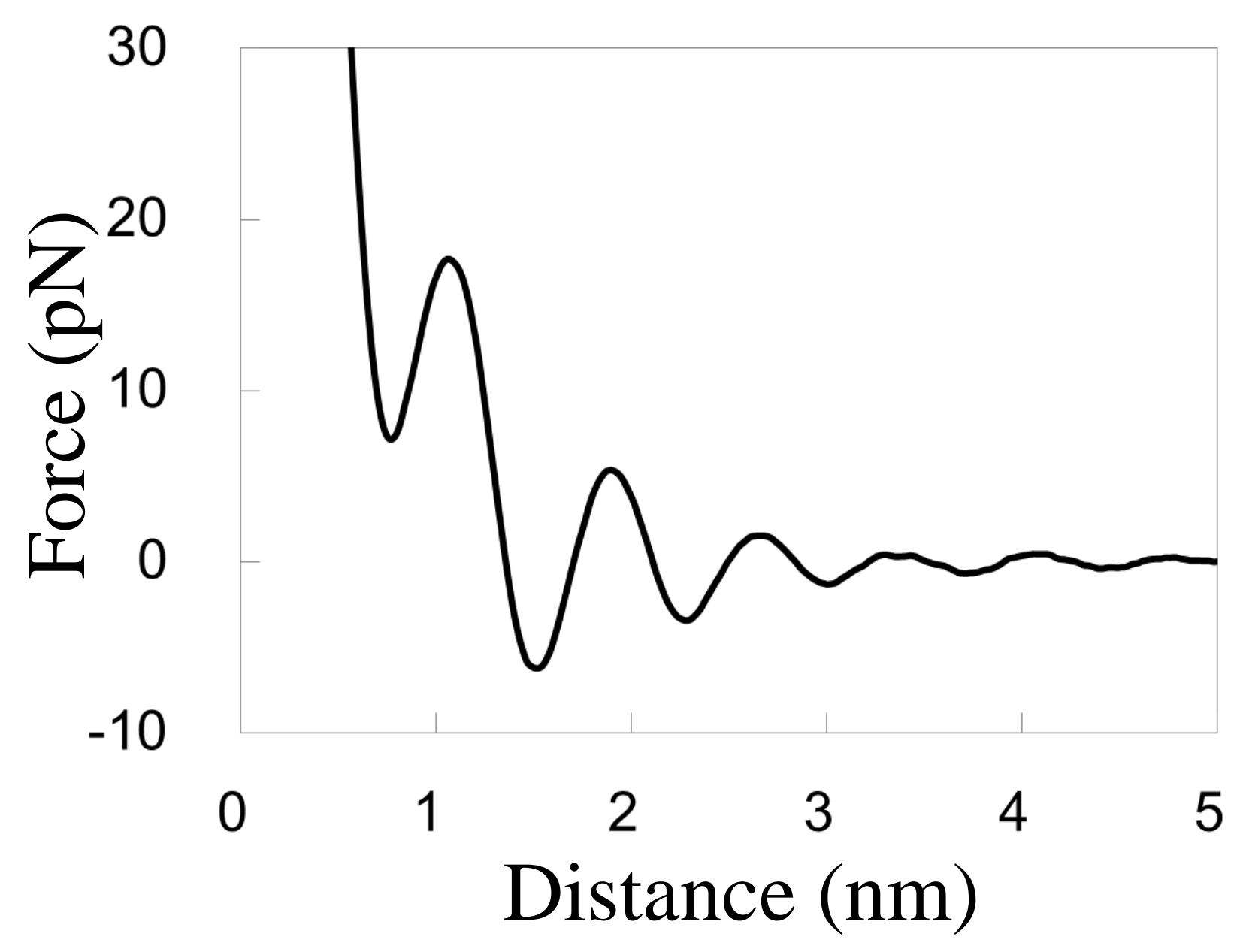

Fig. 1 

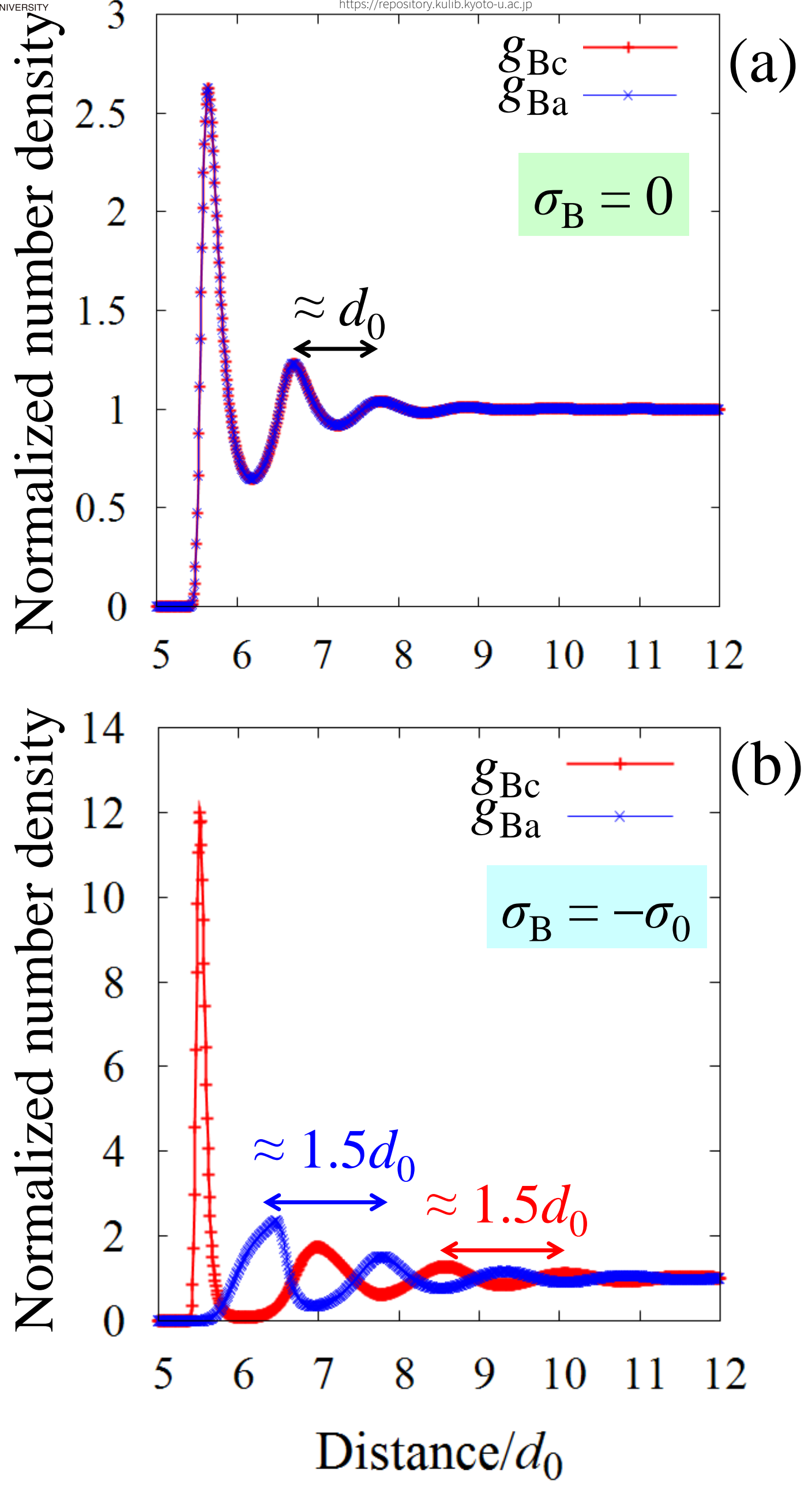

Fig. 2 

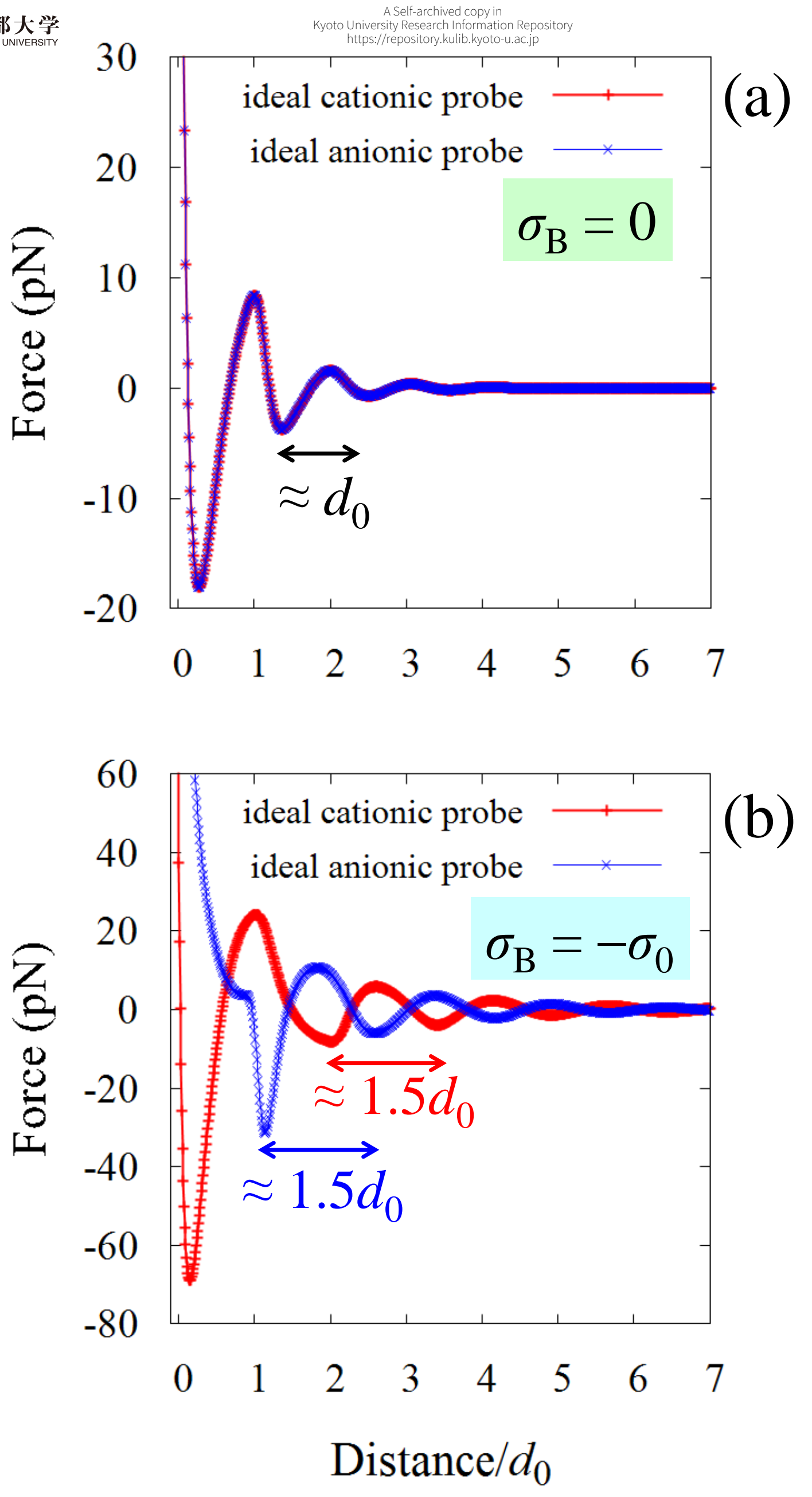

Fig. 3 

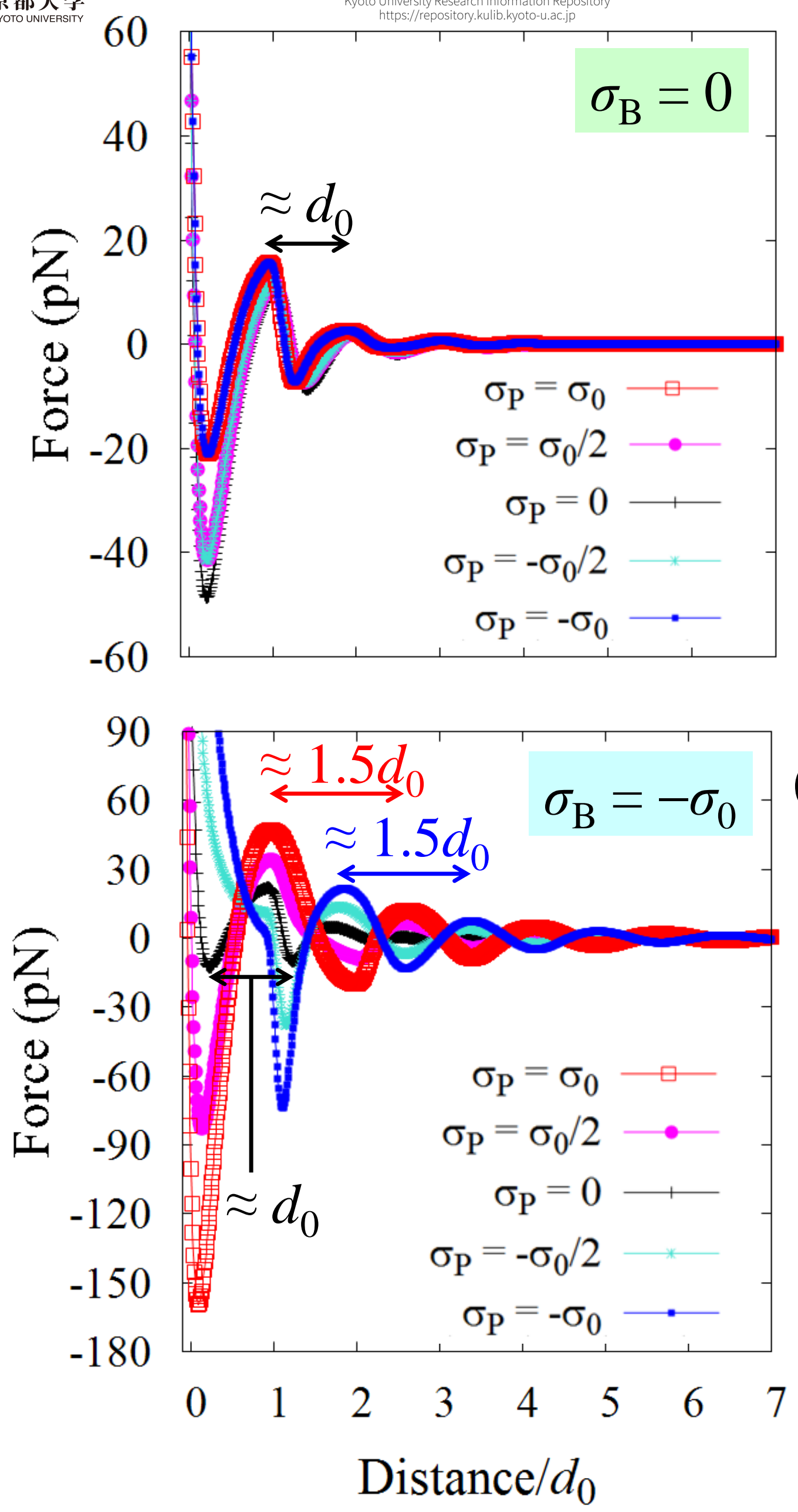

Fig. 4 


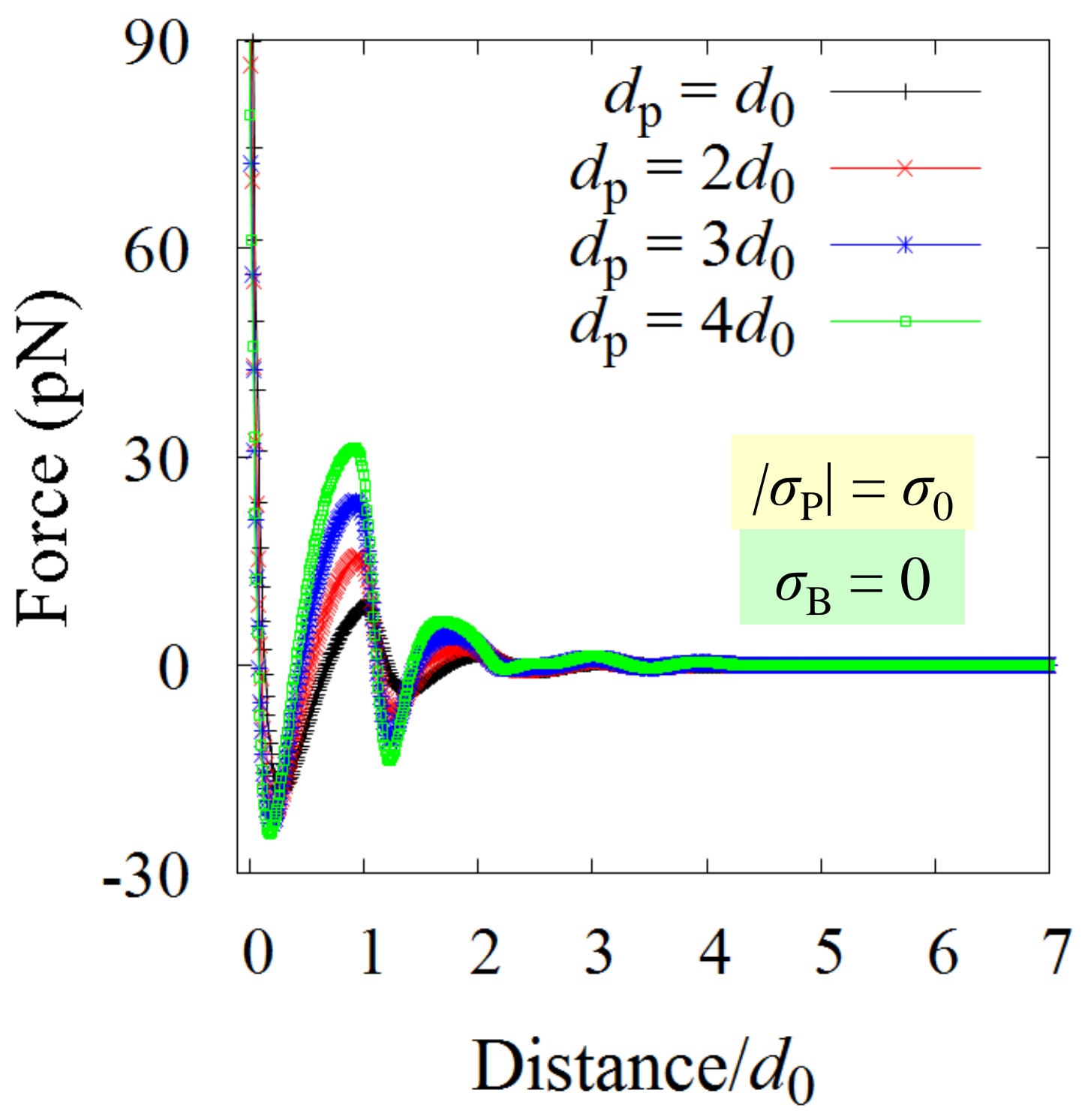

Fig. 5 

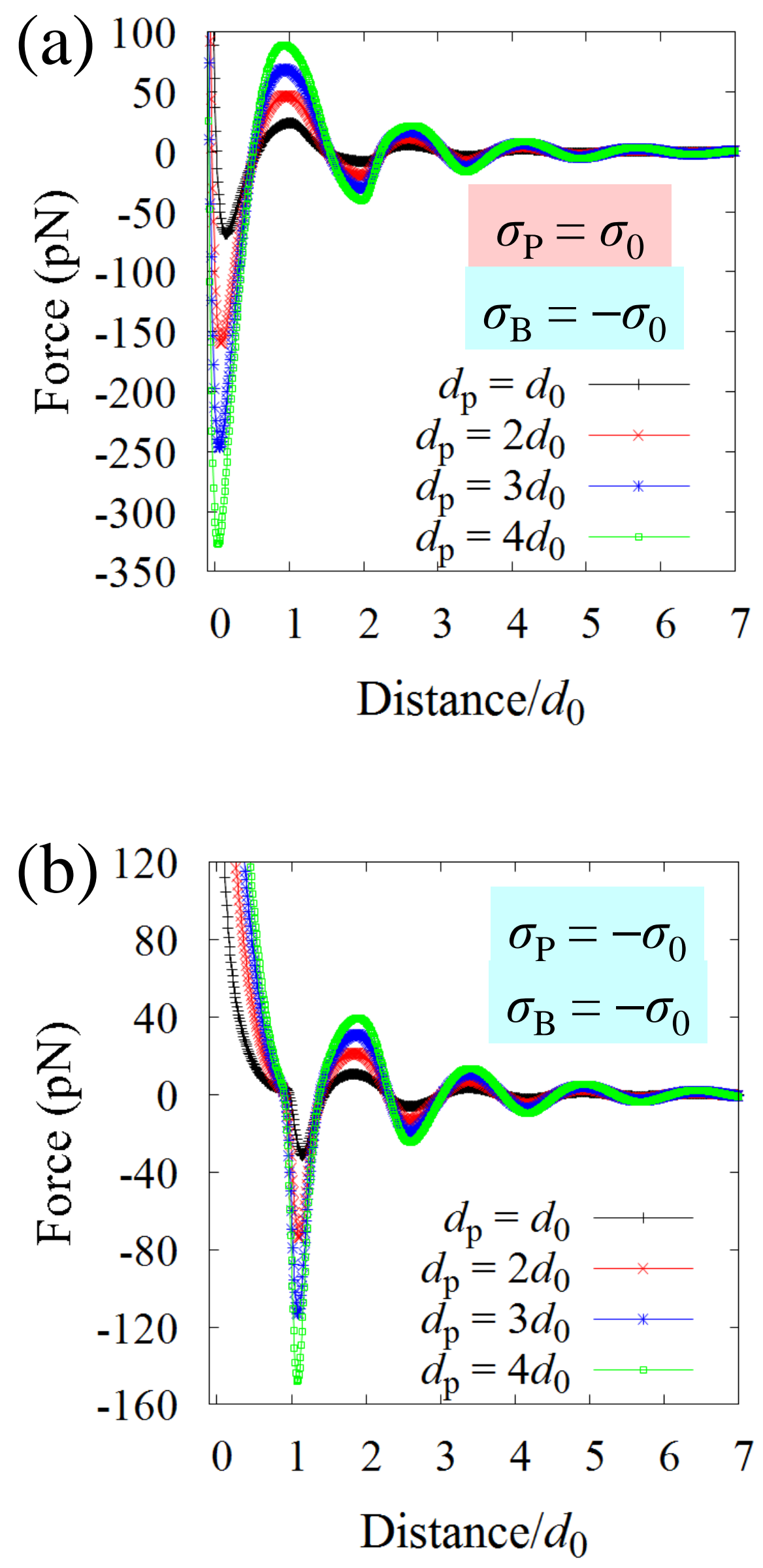

Fig. 6 


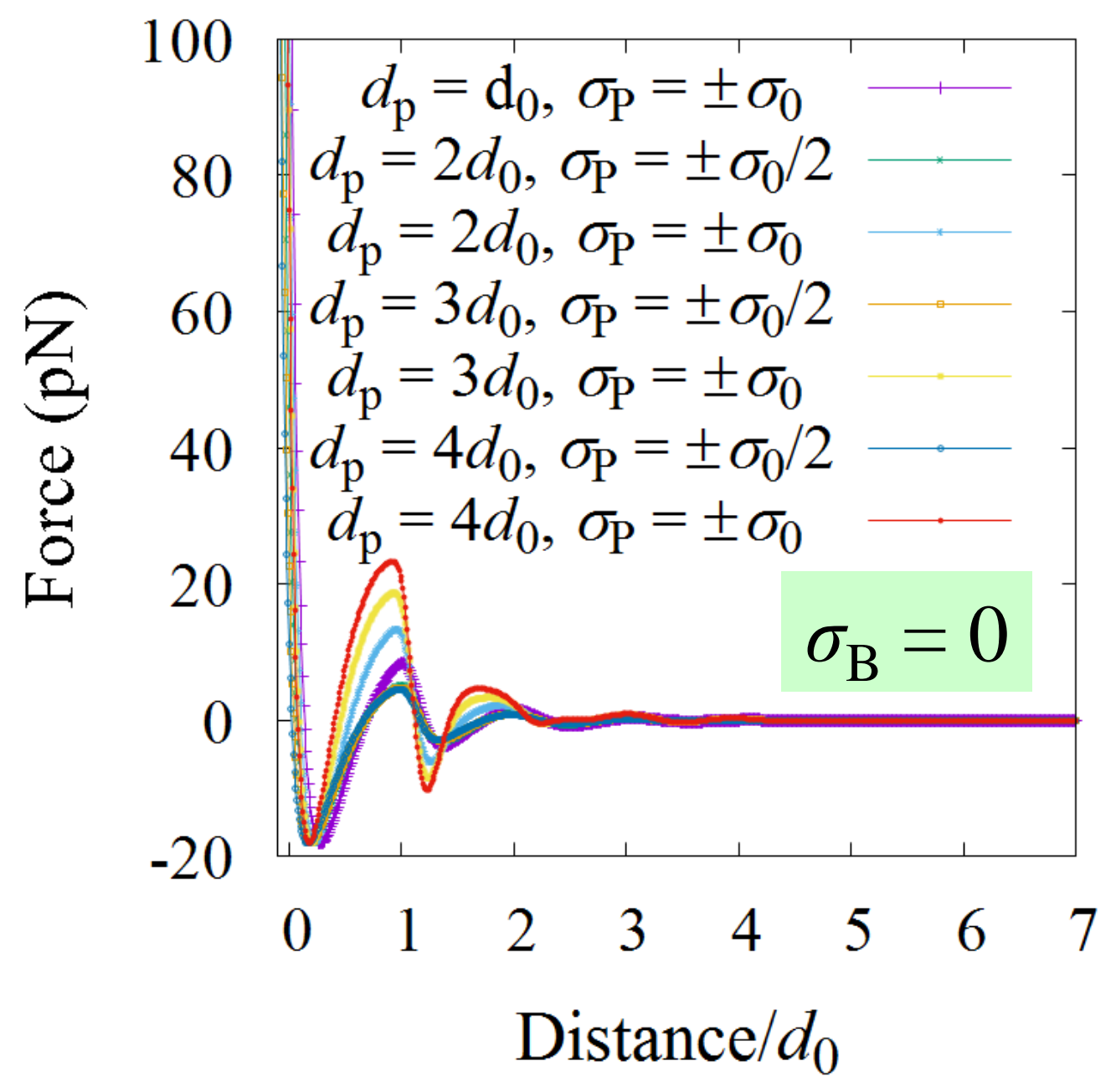

Fig. 7 


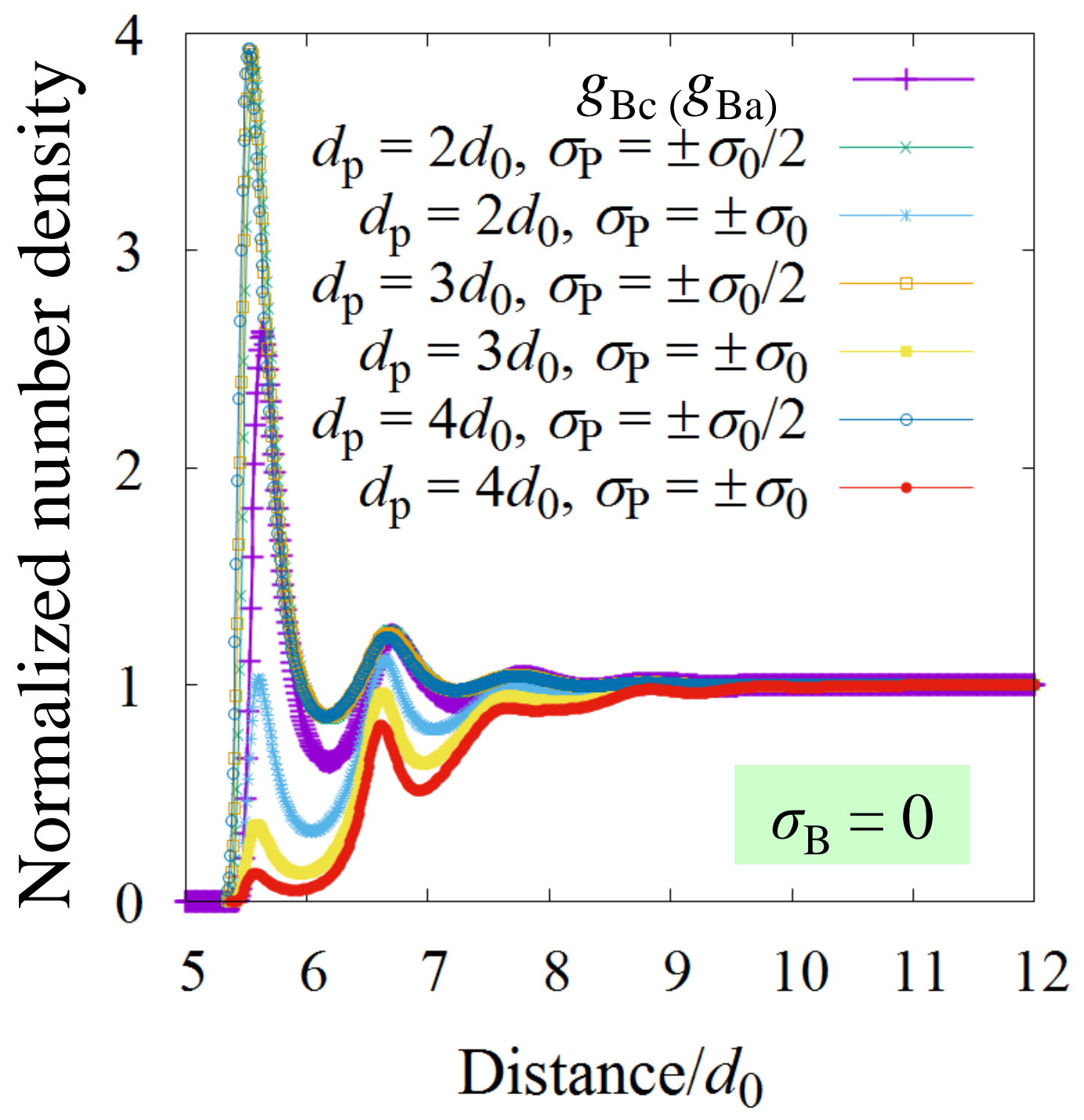

Fig. 9 

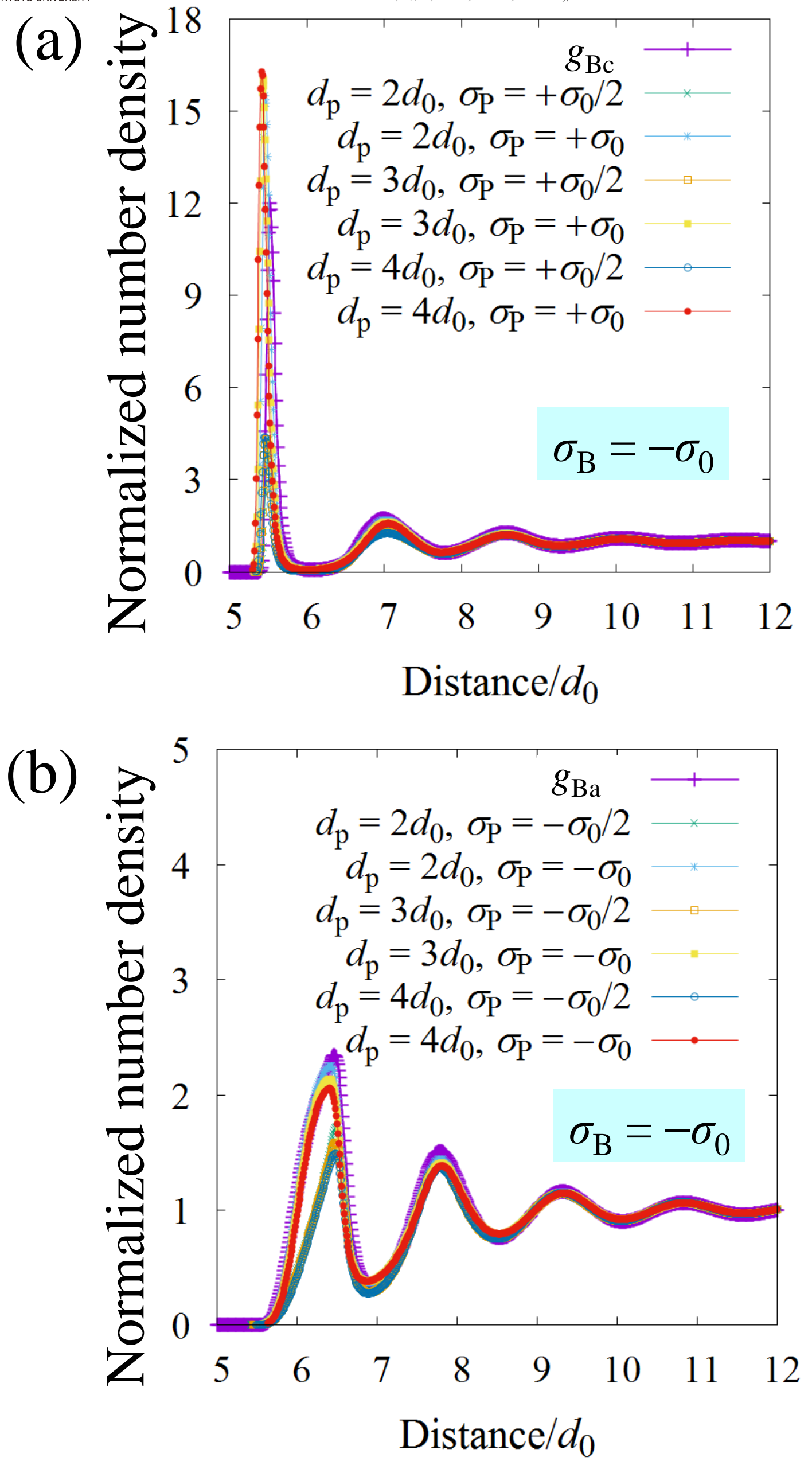

Fig. 10 


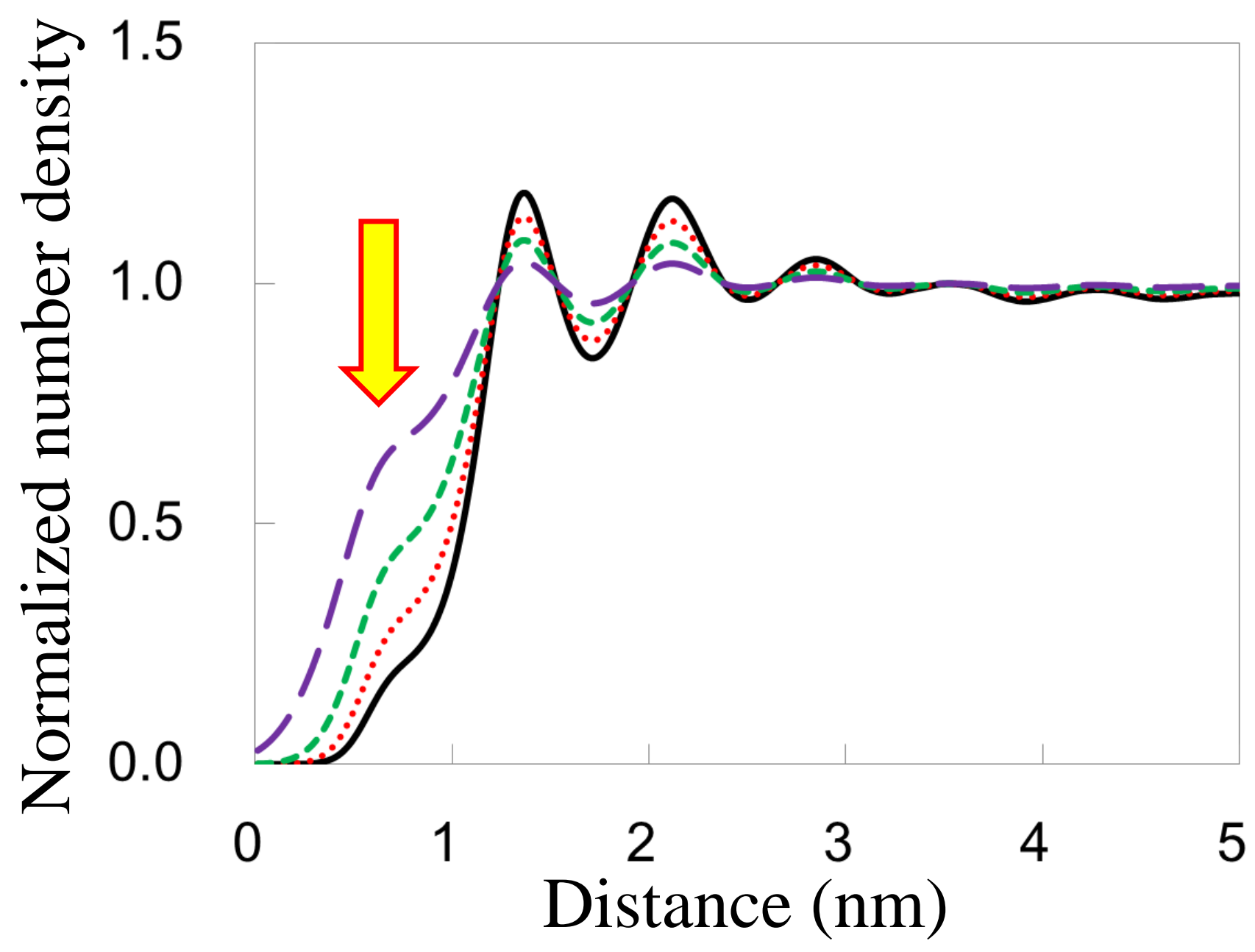

Fig. 11 


\section{Electronic Supplementary Information (ESI)}

\section{Relationship between Force Curve Measured by Atomic Force Microscopy in Ionic Liquid and its Density Distribution on a Substrate}

Ken-ichi Amano, ${ }^{a^{*}}$ Yasuyuki Yokota, ${ }^{\mathrm{b}}$ Takashi Ichii, ${ }^{\mathrm{c}}$ Norio Yoshida, ${ }^{\mathrm{d}}$ Naoya Nishi, ${ }^{a}$ Seiji Katakura, ${ }^{a}$ Akihito Imanishi, ${ }^{e}$ Ken-ichi Fukui, ${ }^{e}$ and Tetsuo Sakka ${ }^{a}$

${ }^{a}$ Department of Energy and Hydrocarbon Chemistry, Graduate School of Engineering, Kyoto University, Kyoto 615-8510, Japan.

E-mail: amano.kenichi.8s@kyoto-u.ac.jp

${ }^{\mathrm{b}}$ Surface and Interface Science Laboratory, RIKEN, Saitama 351 -0198, Japan

c Department of Materials Science and Engineering, Kyoto University, Kyoto, 606-8501, Japan

${ }^{\mathrm{d}}$ Department of Chemistry, Graduate School of Science, Kyushu University, Fukuoka 819-0395, Japan

${ }^{\mathrm{e}}$ Department of Materials Engineering Science, Graduate School of Engineering Science, Osaka University, 1-3 Machikaneyama, Toyonaka, Osaka 560-8531, Japan 


\section{Figure and Tables}

\section{$\mathrm{OZ}-\mathrm{HNC}$ for the ionic liquid}

(1) Calculation of bulk structure of a ionic liquid: Density distribution of cations around a cation, Density distribution of anions around a cation, Density distribution of cations around an anion, Density distribution of anions around an anion.

\section{(2) Calculation of the solvation structures:}

Density distribution of cations around a probe, Density distribution of anions around a probe, Density distribution of cations on a substrate, Density distribution of cations around a substrate.

(3) Calculation of mean force between the substrate and the probe.

If the probe is the ideal one, use Eq. (3), else use both Eq. (3) and Eq. (5).

(The probe surface must be positively or negatively charged.)

\section{(4) Reconstruction:}

Density distribution of cations on a substrate, Density distribution of cations around a substrate.

Fig. S1 Overview of the calculation process. 
Table S1 Values of $C^{*}$ for conditions written in Fig. 7 , where $\sigma_{\mathrm{B}}=0$. The value of $C^{*}$ increases with increase in $d_{\mathrm{P}}$. The value of $C^{*}$ for large $\left|\sigma_{\mathrm{P}}\right|$ is smaller than that for

\begin{tabular}{|c|c|c|}
\multicolumn{3}{|c}{ small $\left|\sigma_{\mathrm{P}}\right|}$. \\
\hline$d_{\mathrm{P}}(\mathrm{m})$ & $\sigma_{\mathrm{P}}\left(\mathrm{C} / \mathrm{nm}^{2}\right)$ & $C^{*}$ \\
\hline $2 d_{0}$ & $\pm \sigma_{0}$ & 0.4356 \\
$2 d_{0}$ & $\pm \sigma_{0} / 2$ & 0.8553 \\
$3 d_{0}$ & $\pm \sigma_{0}$ & 0.3088 \\
$3 d_{0}$ & $\pm \sigma_{0} / 2$ & 0.7944 \\
$4 d_{0}$ & $\pm \sigma_{0}$ & 0.2431 \\
$4 d_{0}$ & $\pm \sigma_{0} / 2$ & 0.7459 \\
\hline
\end{tabular}

Table S2 Values of $C^{*}$ for conditions written in Fig. $8(\mathrm{a})$, where $\sigma_{\mathrm{B}}=-\sigma_{0}$. The value of $C^{*}$ decreases with increase in $d_{\mathrm{P}}$. The value of $C^{*}$ for large $\sigma_{\mathrm{P}}$ is larger than that for small $\sigma_{\mathrm{P}}$.

\begin{tabular}{|c|c|c|}
\hline$d_{\mathrm{P}}(\mathrm{m})$ & $\sigma_{\mathrm{P}}\left(\mathrm{C} / \mathrm{nm}^{2}\right)$ & $C^{*}$ \\
\hline $2 d_{0}$ & $+\sigma_{0}$ & 0.8312 \\
$2 d_{0}$ & $+\sigma_{0} / 2$ & 0.4324 \\
$3 d_{0}$ & $+\sigma_{0}$ & 0.5413 \\
$3 d_{0}$ & $+\sigma_{0} / 2$ & 0.2798 \\
$4 d_{0}$ & $+\sigma_{0}$ & 0.4094 \\
$4 d_{0}$ & $+\sigma_{0} / 2$ & 0.2107 \\
\hline
\end{tabular}

Table S3 Values of $C^{*}$ for conditions written in Fig. 8(b), where $\sigma_{\mathrm{B}}=-\sigma_{0}$. The value of $C^{*}$ decreases with increase in $d_{\mathrm{P}}$. The value of $C^{*}$ for large $\left|\sigma_{\mathrm{P}}\right|$ is larger than that for small $\left|\sigma_{\mathrm{P}}\right|$.

\begin{tabular}{|c|c|c|}
\hline$d_{\mathrm{P}}(\mathrm{m})$ & $\sigma_{\mathrm{P}}\left(\mathrm{C} / \mathrm{nm}^{2}\right)$ & $C^{*}$ \\
\hline $2 d_{0}$ & $-\sigma_{0}$ & 0.8273 \\
$2 d_{0}$ & $-\sigma_{0} / 2$ & 0.4237 \\
$3 d_{0}$ & $-\sigma_{0}$ & 0.5563 \\
$3 d_{0}$ & $-\sigma_{0} / 2$ & 0.2762 \\
$4 d_{0}$ & $-\sigma_{0}$ & 0.4311 \\
$4 d_{0}$ & $-\sigma_{0} / 2$ & 0.2116 \\
\hline
\end{tabular}

